Lösung bilden sich, vielleicht auch primär, neben und im Gleichgewicht mit Jod unterjodige Säure und Hypojodit an der Anode.

3. In neutraler Jodkalium-Losung bleiben während und nach der Elektrolyse kleine Mengen von freiem Jod und Alkali im Gleichgewicht mit Spuren von unterjodiger Säure bestehen, während die Hauptmenge des anfangs entstandenen Hypojodits sofort sich in Jodat verwandelt.

4. Ist die Jodkalium-Lösung stärker alkalisch, so erlangt der durch den anodischen Vorgang im Elektrolyten anfangs ausschliesslich erzeugte Hypojoditsauerstoff eine grössere Beständigkeit, verwandelt sich aber, wenn seine Konzentration ansteigt, immer schneller (ausschliesslich oder wesentlich durch sekundäre Reaktion) in Jodatsauerstoff.

5. Das Hypojodit erreicht hierdurch allmählich eine konstante Konzentration, welche, bei gegebener Versuchsanordnung, durch Stromstärke, Temperatur, den Jodid- und den Alkaligehalt der Lősung beeinflusst wird. Ist diese Konstanz des Hypojodits erreicht, so liefert die Elektrolyse nur noch Jodat.

6. Bei der Elektrolyse sehr verdünnter, alkalischer Jodkaliumlösungen entsteht, zumal an glatter Anode, auch Sauerstoffgas, dessen Menge mit abnehmender Jodidkonzentration steigt und fár den gleichen Wert derselben um so grosser ist, je stärker die Alkalität der Lösung und je hơher Stromdichte und Temperatur sind.
7. Perjodat entsteht bei der Elektrolyse von Jodidlösungen, wenn uberhaupt, so nur in ganz geringer Menge.

Für die elektrolytische Darstellung von Alkalijodaten ${ }^{1}$ ) ist es zweckmässig, folgende Bedingungen innezuhalten: Das zu elektrolysierende Jodkalium wird in mindestens normaler, womöglich aber höherer Konzentration angewandt und die Lösung durch freies Alkali etwa 0,5 -normal gemacht und auf je $100 \mathrm{ccm} \mathrm{mit}$ $0,2 \mathrm{~g}$ Kaliumchromat versetzt. Als Anode dient ein blankes, senkrecht hängendes Platinblech, welches im Abstande von I,5 bis $2 \mathrm{~cm}$ zwischen zwei parallel angeordneten Gitterkathoden aus Platindraht sich befindet; die Elektroden stehen einige Centimeter vom Boden des Gefässes ab. Man elektrolysiert unter dauerndem Umrühren des Elektrolyten bei einer anodischen Stromdichte von etwa o,o I Amp/qcm, bis die Klemmenspannung über 2 Volt steigt, und an der Anode reichlichere Sauerstoffentwicklung beginnt, und entfernt jetzt das am Boden ausgeschiedene Jodat von der Lösung, welche man bei weiteren Elektrolysen immer wieder als Lösungsmittel verwendet. Will man eine bestimmte Menge Jodid möglichst weitgehend aufarbeiten, so setzt man die Elektrolyse so lange fort, bis etwa $10 \%$ über die theoretisch erforderliche Strommenge verbraucht sind.

Dresden, im Januar I903. (Eingegangen: 30 . Januar.)

r) Vergl. auch Elbs, Uebungsbeispiele, S. 3o.

\title{
BEMERKUNGEN ZUR ELEKTROCHEMIE DER ÜBERMANGANSÄURE ${ }^{1}$ ).
} Von $J$.K. H. Inglis, M. A., B. Sc.

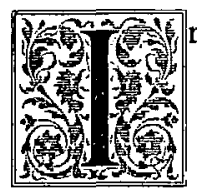
Anbetracht des häufigen Gebrauchs von Permanganaten in chemischen Laboratorien zu Oxydationszwecken ist es von grosser Wichtigkeit, den Einfluss der Anwesenheit anderer Substanzen auf die Oxydationskraft zu kennen. Empirische Regeln sind vielfach aufgestellt worden und werden befolgt, z. B. ist das Hinzufügen von Manganosalzen vorgeschrieben, wenn Ferrosalze bei Gegenwart von Chloriden titriert werden

I) Nach eingesandtem Manuskript übersetz.t. H. D. sollen, um eine Oxydation des Chlorides zu Chlor zu verhindern ${ }^{1}$ ). Quantitative Messungen uber den Einfluss der Manganosalzkonzentration sind jedoch nie gemacht worden, da konstante Resultate nicht erhalten werden konnten.

Nach Ostwald kann die Oxydationskraft durch Messung des Oxydationspotentials der Substanz bestimmt werden; Bancroft ${ }^{2}$ ) und

2) Pogg. Ann. 118, 45, 119, 225.

3) Zeitschr. f. physikal. Chemie 10, $3^{87}$. 
Neumann ') haben eine grössere Anzahl solcher Messungen gemacht. Zuerst wurde angenommen, dass das Oxydationspotential unabhängig von der Konzentration sei; die richtige Beziehung zwischen beiden hat Peters ${ }^{2}$ ) aufgestellt. Im Falle des Permanganates schienen jedoch alle quantitativen Messungen $z u$ versagen, wenn auch eine rohe quantitative Verifizierung der Theorie Crotogin ${ }^{3}$ ) gelang. Neuerdings hat Haber ${ }^{4}$ ) die vorliegenden Messungen gesichtet und sie mit den Forderungen der Thermochemie verglichen. Nun entspricht, wie Luther ${ }^{5}$ ) gezeigt hat, ein ganz bestimmter Potentialwert jedem Vorgang, der bei der Reduktion eines Oxydationsmittels eintreten kann, und es lassen sich einfache Gleichungen aufstellen, die die Beziehung zwischen den Potentialen, die zu diesen verschiedenen Vorgängen gehören, angeben. Da Uebermangansăure zu Mangansuperoxyd und zu Manganosalz reduziert werden kann, existieren zwei Potentiale, von denen das dem ersten Vorgange entsprechende numerisch den grösseren Wert hat. Bancroft erhielt den Wert 1,76 Volt, und nahm an, dass dieses Potential der Reaktion

$$
\mathrm{MnO}_{4}+8 \mathrm{H}^{\cdot}=\mathrm{Mn}^{\cdot}+{ }_{4} \mathrm{H}_{2} \mathrm{O}+5 \oplus
$$
entspreche.

Zweck dieser Untersuchung ist nun, zu prufen, ob dies richtig ist oder ob die Messung Bancrofts nicht die Reaktion:

$$
\mathrm{MnO}_{4}{ }^{\prime}+{ }_{4} \mathrm{H}^{\circ}=\mathrm{MnO}_{2}+2 \mathrm{H}_{2} \mathrm{O}+3 \oplus
$$
betrifft

In Beziehung zu den beiden Gleichungen steht eine dritte:

$$
\mathrm{MnO}_{2}+{ }_{4} \mathrm{H}^{\cdot}=\mathrm{Mn} \cdot{ }^{*}+2 \mathrm{H}_{2} \mathrm{O}+2 \oplus \text {, }
$$

die genau durch Tower ${ }^{6}$ ) untersucht wurde; er fand das diesem Vorgang entsprechende Potential zu I,63 Volt. Unter Benutzung seiner Resultate konnen wir die den Reaktionen I und 2 entsprechenden Potentiale nach Luthers Formel ${ }^{7}$ ) berechnen, wenn wir den Wert für eines derselben kennen. Deshalb wurde nach Wiederholung einiger Messungen von Tower die Reaktion 2 unter Aenderung der Konzentration
I) ib. 14, 210.
2) ib. 26, 193 .
3) Zeitschr. f. anorgan. Chemie 24, 225.
4) Zeitschr. f. Elektrochemie 7, I043.
5) Zeitschr. f. physikal. Chemie 34, $4^{88}, 36,3^{85}$.
6) Zeitschr. f. physikal. Chemie 18, I7.
7) 1. c.

studiert und wurden gleichzeitig die entsprechenden Potentialänderungen gemessen.

Die Formel von Peters lautet:

$$
P_{2}=P_{2}^{\prime}+\frac{R T}{3 F} \ln \frac{C_{M_{n} O_{4}} \cdot C^{4} H^{*}}{C_{M_{n} O_{2} \cdot C^{2} H_{2} O}},
$$

in der $R$ die Gaskonstante, $T$ die absolute Temperatur und die indizierten $C$ die Konzentrationen der im Index stehenden Stoffe sind, und $P_{2}^{\prime}$ das Potential, wenn alle $C=\mathrm{I}$ sind. $C_{H_{2} O}$ kann als konstant betrachtet werden, da die Lobsungen verdünnt waren; da $\mathrm{MnO}_{2}$ als fester Niederschlag auf der Elektrode vorhanden ist, ist auch $C_{\mathrm{MnO}_{2}}$ eine Konstante. Bei Zimmertemperatur lautet obige Formel also:

$$
P_{2}=P_{2}{ }^{\prime}+\frac{0,05^{8}}{3} \log C_{\mathrm{MnO}_{4}{ }^{\prime}} \cdot \mathrm{C}^{4} \mathrm{H}^{{ }^{*}},
$$

eine quantitative Beziehung, die sich prafen lăsst. In ăhnlicher Weise können auch die übrigen Reaktionen geprüft werden. Für die Reaktion 3 sind die entsprechenden Messungen schon von Tower gemacht worden; doch um Interpolation seiner Resultate zu vermeiden, wurden sie wiederholt, und zwar mit denselben Konzentrationen, die bei den Messungen für I und 2 benutzt wurden.

\section{Versuchsresultate.}

Für sămtliche Potentialmessungen wurde die Poggendorfsche Kompensationsmethode benutzt; ein Akkumulator diente als Arbeitselement an der Wheatston eschen Brücke, so dass an den Enden des Brückendrahtes 2 Volt waren. Ein Lippmannsches Kapillarelektrometer war Nullinstrument, der Akkumulator wurde gegen ein Kadmium-Normalelement ( $\mathrm{I}, \mathrm{org}$ Volt) geaicht. Als Normale diente eine DecinormalKalomelelektrode (0,6I6 Volt); sie wurde an die zu messende Elektrode mittels eines Hebers, gefüllt mit der entsprechenden Lösung und in gesättigte $\mathrm{KCl}$-Losung tauchend, angeschlossen. Die E. M.K. konnte auf 0,00 I Volt genau gemessen werden, doch sind die Versuchsfehler etwas grösser anzunehmen.

Zuerst wurde das Potential einer $\mathrm{MnO}_{2}$ Elektrode gegen eine mit $\mathrm{MnSO}_{4}$ versetzte Schwefelsäurelösung gemessen. Die $\mathrm{MnO}_{2}$-Elektroden bestanden aus Platinblech, auf welchem elektrolytisch $\mathrm{MnO}_{2}$ niedergeschlagen war; sie wurde gewaschen und als Anode in Schwefelsăure der elektrolytischen Formierung unter- 
worfen, um etwa anhaftendes Manganosalz zu oxydieren. Die erhaltenen Resultate sind:

Tabelle I.

I $\mathrm{Mol} \mathrm{MnSO}_{4}$ in 100, 1000, 10000 Liter ergab für $\mathrm{r} \mathrm{Mol}_{2} \mathrm{SO}_{4}$

$$
\text { in } 20 \text { Liter I,55I, I,574, I,602 Volt, }
$$$$
\text { "200 "I,437, I,467, I,490" }
$$

Dię Resultate sind vollkommen gleichmăssig und gehorchen der Formel:

$$
P_{3}=P_{3}{ }^{\prime}+0,029 \log \frac{C^{4} H^{*}}{C_{M n^{*}}} \text {. }
$$

Das Potential, welches zu dem Vorgang $\mathrm{MnO}_{4}{ }^{\prime} \rightarrow \mathrm{MnO}_{2}$ gehört, muss mit Hilfe von $\mathrm{MnO}_{2}$ in einer sauren Lösung von Permanganat gemessen werden. Da Permanganat empfindlich ist gegen Staub, der aus der Luft hineinkommt, so wurden alle Messungen in einem geschlossenen Gefäss ausgefuhrt, welches drei Elektroden enthielt und mittels eines Hebers mit der Normalelektrode verbunden war.

Stets wurde das Potential mehrere Mal nach Verlauf je mehrerer Stunden gemessen, und zwar dasjenige aller drei Elektroden; aus den nach Konstantwerden gewonnenen Zablen wurde das Mittel genommen. In der ersten Versuchsserie wurde die Konzentration des Permanganats konstant gehalten (I Mol in roo Liter) und die Konzentration der Schwefelsäure geăndert. Tabelle 2 giebt die Resultate wieder.

Tabelle 2.

I $\mathrm{Mol} \mathrm{KMnO}_{4}$ in roo, roo, roo Liter, I " $\mathrm{H}_{2} \mathrm{SO}_{4}$ " 4, 20, 100 , Potential, gemessen $\mathrm{I}, 8 \circ 3, \mathrm{r}, 77 \mathrm{I}, \mathrm{I}, 73 \mathrm{I}$, korrigiert $\quad 1,816, \quad 1,776, \quad I, 73^{x}$.

In der năchsten Versuchsreihe (Tabelle 3) wurde die Schwefelsäurekonzentration konstant gehalten (I Mol in 2o Liter) und die des Permanganats variiert.

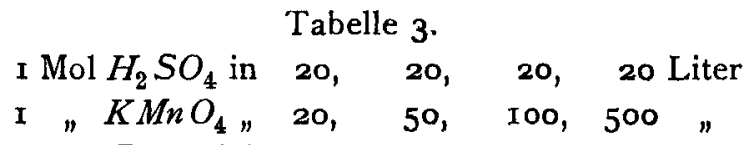
Potential $1,787,1,781,1,771,1,761$.

Trăgt man die Resultate der Tabelle 3 in ein Koordinatensystem ein, in welchem man statt der Konzentrationen den Logarithmus derselben wahlt, so ergiebt sich, dass die vier Punkte auf einer vollkommen geraden Linie liegen. Es berechnet sich aus dieser geraden Linie die Gleichung:

$$
P_{2}=P_{2}^{\prime \prime}+0,019 \log C_{M_{n} \mathrm{O}^{\prime \prime}} \text {, }
$$

wăhrend Reaktion 2 die Formel:

$$
P_{2}=P_{2}{ }^{\prime}+\frac{0,5^{8}}{3} \log C_{M_{n} O_{4}} \cdot C^{4} H^{*}
$$

verlangt.

Die Resultate der Tabelle 3 sind also vollkommen mit der Formel in Uebereinstimmung, wenn $\log \mathrm{C}^{4} H^{*}$ als Konstante in den Wert $P_{2}$ einbezogen wird.

Was Tabelle 2 anlangt, so ist zu berücksichtigen, dass allemal die Summe des eigentlichen Elektrodenpotentials und des Potentials an der Berührung der Säure mit der konzentrierten $\mathrm{KCl}$-Lösung gemessen wird; das Potential an der Berührungsstelle von gesättigter $\mathrm{KCl}$ - und Decinormal-Elektrode kann vernachlässigt werden. Nun ändert sich das Potential "Săure | ges. $\mathrm{KCl}^{\prime \prime " ~ m i t ~ d e r ~ S a ̈ u r e k o n z e n t r a t i o n . ~}$ Nach der Formel von $\mathrm{Planck}^{1}$ ) finden sich die Werte:

I $\mathrm{Mol} \mathrm{H}_{2} \mathrm{SO}_{4}$ in 4, 2o, roo Liter, ergiebt das Potential zwischen $\mathrm{H}_{2} \mathrm{SO}_{4}$ und $\mathrm{KCl}$ o, o $3,0,005, \sim 0$.

Diese Werte mússen also den gemessenen Werten der Tabelle 2 zuaddiert werden. Die so gewonnenen, „korrigierten“ Werte der Tabelle 2 zeigen eine mittlere Abnahme des Potentials um 0,06 Volt, wenn die Verdünnung um das Zehnfache wächst. Bei zehnfacher Verdünnung nimmt aber die $H^{\circ}$-Ionenkonzentration weniger $a b$, als $x:$ ro wegen des grosseren Dissociationsgrades bei grösserer Verdunnung. Man erhălt so annähernd:

$$
P_{2}=P_{2}^{\prime}+0,065 \log C_{H^{\prime}} \text {, }
$$

was annähernd mit dem theoretischen Wert 0,077 stimmt. Es ergiebt sich, dass das hier gemessene Potential der Reaktion 2 entspricht.

Wir können nunmehr Luthers Formel anwenden fur die Berechnung des Potentials der Reaktion:

$$
\mathrm{MnO}_{4}{ }^{\prime} \rightarrow \mathrm{Mn} \cdot .
$$

Nennen wir dieses Potential $P_{1}$, so ergiebt sich:

$$
\begin{gathered}
P_{1} \cdot 5 F=P_{y} \cdot 3 F+P_{3} \cdot 2 F \\
P_{1}=\frac{3 P_{2}+2 P_{3}}{5} .
\end{gathered}
$$

I) Wied. Annal. d. Physik 40, $56 \mathrm{r}$. 
Für eine Lösung:

I $\mathrm{Mol} \mathrm{H}_{2} \mathrm{SO}_{4}$ in 20 Liter und I $\mathrm{Mol} \mathrm{MnSO}_{4}$ in 10000 Liter ist $P_{3}=\mathrm{I}, 602$,

für I Mol $\mathrm{H}_{2} \mathrm{SO}_{4}$ in 20 Liter und I $\mathrm{Mol} \mathrm{KMnO}_{4}$ in 100 Liter ist $P_{2}=\mathrm{I}, 77 \mathrm{I}$,

also für $1 / 20 \mathrm{Mol} \mathrm{H}_{2} \mathrm{SO}_{4}, 1 / 10000 \mathrm{Mol} \mathrm{MnSO}_{4}$, $1 / 100 \mathrm{Mol} \mathrm{KMnO}_{4}$ in I Liter ergiebt sich:

$$
P_{1}=\frac{3 \cdot 1,77 \text { I }+2 \cdot 1,602}{5}=1,703
$$

I,703 Volt müsste also eine Platinelektrode in dieser gemischten Lösung aufweisen.

Stellt man solch eine Lösung her, so bildet sich ein brauner Niederschlag, der aus einem hydratisierten Peroxyd besteht und gleichzeitig hydratisiertes (?) Manganooxyd enthält. Dadurch ändert sich aber wieder die Konzentration, und man kann nicht erwarten, den berechneten Potentialwert zu erhalten. Ich fand, dass das Potential zuerst 1,716 war und innerhalb mehrerer Tage auf $1,74^{\circ}$ stieg. Eine zweite Lösung mit nur $1 / 10$ der oben genannten Manganosulfatmenge, die der Berechnung nach ein Potential von I,7 I 4 haben sollte, gab ebenfalls einen schwachen Niederschlag von $\mathrm{MnO}_{2}$, und das Potential stieg von 1,700 auf $1,7 \mathrm{I} 7$ in vier Stunden und dann weiter auf 1,728 in drei Tagen. Demnach stört die Entstehung des Niederschlages die quantitativen Beziehungen, da ein Mehr an Manganosalz statt einer Abnahme des Potentials eine Zunahme desselben hervorruft. Die hier stattfindende Reaktion formuliert Ostwald 1 ): $2 \mathrm{MnO}_{4}+{ }_{3} \mathrm{Mn}^{\prime \prime}+{ }_{2} \mathrm{H}_{2} \mathrm{O}=5 \mathrm{MnO}_{2}+{ }_{4} \mathrm{H}^{\circ}$, wobei das $\mathrm{MnO}_{2}$ vermutlich durch Hydrolyse eines Manganisalzes entsteht. Diese Reaktion findet so lange statt, bis Gleichgewicht erreicht ist. Sie kann zerlegt werden in:

$$
\begin{gathered}
2\left(\mathrm{MnO}_{4}^{\prime}+{ }_{4} \mathrm{H}^{\circ}=\mathrm{MnO}_{2}+2 \mathrm{H}_{2} \mathrm{O}+3 \oplus\right) \\
3\left(\mathrm{MnO}_{2}+{ }_{4} \mathrm{H}^{\circ}=\mathrm{Mn}^{\prime}+2 \mathrm{H}_{2} \mathrm{O}+2 \oplus .\right)
\end{gathered}
$$

Die Reaktion verläuft also so lange, bis die beiden Potentiale, die diesen Reaktionen entsprechen, gleich geworden sind, sowohl untereinander, wie auch dem Potential der Reaktion:

$$
\mathrm{MnO}_{4}{ }^{\prime} \rightarrow \mathrm{Mn}{ }^{\prime} \text {. }
$$

Deshalb müsste in der resultierenden Lösung eine Platinelektrode dasselbe Potential haben, wie eine $\mathrm{MnO}_{2}$-Elektrode. Dies ist aber nach angestellten Versuchen nicht der Fall, wofür der Grund leicht zu finden ist. Da die Konzen-

1) Grundlinien der anorgan. Chemie, 606. tration der $M n^{*}$-Ionen sehr klein ist, ist die Platinelektrode äusserst polarisierbar und giebt deshalb ein zu kleines Potential.

Aus den für die Reaktionen 2 und 3 gemessenen Potentialen kann man die endgültige Konzentration des $M n^{*}$ berechnen. Wenn man zu einer Lösung, die $1 / 20$ molar in Bezug auf $\mathrm{H}_{2} \mathrm{SO}_{4}$ und $1 / 100$ molar in Bezug auf $\mathrm{KMnO}_{4}$ ist, nur eine kleine Menge $\mathrm{MnSO}_{4}$ hinzufügt, so wird die Konzentration von $\mathrm{H}_{2} \mathrm{SO}_{4}$ und von $\mathrm{KMnO}_{4}$ praktisch dieselbe bleiben. Nun hat eine $\mathrm{MnO}_{2}$-Elektrode in solcher Lösung das Potential I,77I, wenn sie die Reaktion $\mathrm{MnO}_{4}{ }^{\prime}$ $\rightarrow \mathrm{MnO}_{2}$ anzeigt. Dasselbe Potential muss sie haben, wenn sie die Reaktion:

$$
\mathrm{MnO}_{2} \rightarrow \mathrm{Mn} \cdot \cdot
$$

misst. Nun ist in einer $1 / 20$ molaren $\mathrm{H}_{2} \mathrm{SO}_{4}$ das Potential I,602, wenn die $M n$ " - Ionen die Konzentration Io $^{-4}$ molar haben. Deshalb ergiebt sich die'Konzentration für das Potential I,77 I aus der Gleichung:

$$
\begin{aligned}
\mathrm{I}, 77 \mathrm{I}-\mathrm{I}, 602 & =0,029 \log \frac{10^{-4}}{x} \\
\log x & =-\mathrm{ro} \\
x & =\mathrm{Io}^{-10} .
\end{aligned}
$$

Wir können nunmehr begreifen, warum das Potential einer Platinelektrode in einer sauren Lősung von Kaliumpermanganat, die Manganosalze enthält, nicht gemessen werden kann. Denn sie ist höchst polarisierbar, und es findet eine Reaktion statt, die praktisch alle Manganoionen aus der Lösung entfernt. Da nun die Konzentration des Manganosalzes in der Grössenordnung $10^{-10}$ sich bewegt, so genügt eine sehr kleine Menge oxydabler Substanz, das Gleichgewicht enorm $z u$ verschieben. Und da ferner die Konzentration der Manganionen so klein ist, wird die Elektrode sehr polarisierbar, und das Gleichgewicht wird während der Messung fortwährend verschoben, und da schliesslich das Gleichgewicht verschieden liegt, je nach den verschiedenen Anfangskonzentrationen, kann man nicht erwarten, Werte zu bekommen, die mit der Formel übereinstimmen.

Es erfolgt das Resultat, dass quantitative Zahlen für das Oxydationspotential $\mathrm{MnO}_{4}{ }^{\prime} \rightarrow \mathrm{Mn}{ }^{*}$ nur durch Berechnung nach der Lutherschen Formel erhalten werden können.

Die obige Berechnung der Konzentration des im Gleichgewicht belindlichen $M n^{*}$-Ions ist 
von Interesse für die Erscheinungen, die bei einer Titration mittels Permanganat auftreten können. Man bemerkt nämlich häufig, dass der erste Tropfen des zugesetzten Permanganats eine dauernde Farbe hervorruft; wird aber etwas Manganosalz vorher zugesetzt, so verschwindet die Farbe sehr rasch, und die Titration lässt sich glatt ausführen. Dies bedeutet, dass die $\mathrm{MnO}_{4}{ }^{\prime}$-Ionen von selbst nur sehr langsam (vielleicht auch gar nicht) oxydieren können. Erst wenn ein Manganisalz oder ein Salz des vierwertigen Mangans in der Lơsung ist, kann die Reaktion vor sich gehen.

Die angefuhrten Versuche wurden im Chemischen Laboratorium der Universität Edinburgh ausgeführt, und ich möchte an dieser Stelle dem Direktor desselben, Herrn Professor Dr. Crum-Brown, für sein freundliches Interesse meinen besten Dank aussprechen.

(Eingegangen: Io. Februar.)

\section{ÜBER DIE ELEKTROLYSE VON GESCHMOLZENEM ÄTZNATRON. SCHLUSSWORT AN HERRN LORENZ. \\ Von $M$. Le Blanc und $J$ Brode.}

Wir waren und sind auch noch der Meinung, dass wir in unserer Entgegnung ${ }^{1}$ ) an Herrn Lorenz unsere Ansicht bezüglich der Arbeit von Herrn Sacher klar dargelegt und begründet haben. Herr Lorenz wirft uns nun neuerdings ${ }^{2}$ ) unrichtige Behauptungen und Widersprüche vor. Um nicht durch Schweigen den Anschein zu erwecken, als ob wir die Berechtigung dieser Vorwürfe anerkennen, müssen wir noch einmal das Wort nehmen, wollen uns aber auf das Notwendigste beschränken.

Wir stellen folgende Stellen nebeneinander. In unserer Hauptarbeit ${ }^{3}$ ) sagen wir: „Die NernstGla sersche Modifikation der Methode zurMessung von Zersetzungsspannungen mit kleiner (Versuchs-) und grosser (Arbeits-) Elektrode setzt selbstverständlich voraus, dass an der grossen Elektrode sich ein ganz bestimmter Vorgang während des ganzen Verlaufes der Messungen vollzieht und infolgedessen ihr Potential vollständig konstant bleibt. Glaser benutzte dazu ein grosses, mit Luftsauerstoff beladenes, platiniertes Platinblech von solcher Kapazität, dass sich auch bei Stromdurchgang sein Potential nicht merklich änderte. Lägen die Verhältnisse bei Sacher ebenso wie bei Glaser, dann müsste, da er als anodischen Zersetzungspunkt für die Entladung der $O H^{\prime}$ lonen I,3 I Volt und als kathodischen für die Entladung der $\mathrm{Na}$-Ionen 2,06 Volt bezeichnet, fär die Zersetzungsspannung des Natriumhydroxydes .... sich die Summe beider Werte
1) Diese Zeitschrift 8, 939, 1902 .
2) Ebenda 9, 155, I903.
3) Ebenda 8, 704, I902.

gleich 3,37 Volt ergeben, während es thatsächlich für diesen Vorgang sicherlich keiner höheren Spannung als 2,2 Volt bedarf. Sacher hat eben .... nicht beachtet, dass seine Arbeitselektrode bei Benutzung als Anode zu einer Sauerstoff-, bei Benutzung als Kathode zu einer Wasserstoffelektrode polarisiert wurde."

Herr Lorenz lässt uns in seiner letzten Entgegnung (S. I57 dieser Zeitschrift) dagegen folgendes sagen: „.... Hiergegen haben nun Le Blanc und Brode eingewendet, dass dies unzutreffend sei. Sacher habe übersehen, dass die grossen Glaserschen Elektroden konstant mit Satuerstoff beladen waren, während seine eigenen grossen Elektroden in beiden Fällen ein verschiedenes Potential besassen. Er hätte daher, um zu dem Wert der Zersetzungsspannungzu gelangen, seine Einzelwerte addieren müssen, wodurch die viel $z u$ hohe Spannung von 3,37 Volt herauskäme....".

Dies findet Herr Lorenz, und wir mit ihm, sehr widerspruchsvoll; er sucht dann unter missverständlicher Auslegung eines Satzes in dem Lehrbuche des einen von uns eine Erklärung, wie wir zu dieser Unklarheit gekommen seien, und entwickelt dann seine eigene Ansicht. Wir müssen es ablebnen, dass Herr Lorenz die Widersprüche, die er selbst erst schafft und dann widerlegt, uns zur Last legt.

Auch dem, was wir in Bezug auf die Brauchbarkeit von Eisen- und Nickelelektroden in unserer letzten Entgegnung gesagt haben, haben wir eigentlich nichts hinzuzufügen. Wir wollen nur gegenuber dem Ausspruch von Herrn 